\title{
TOEPLITZ ALGEBRAS OF CORRESPONDENCES AND ENDOMORPHISMS OF SUMS OF TYPE I FACTORS
}

\author{
PHILIP M. GIPSON
}

\begin{abstract}
It is a well-known fact that endomorphisms of $B(H)$ are intimately connected with families of mutually orthogonal isometries, i.e. with representations of the so-called Toeplitz $C^{*}$-algebras. In this paper we consider a natural generalization of this connection between the representation theory of certain $C^{*}$-algebras associated to graphs and endomorphisms of certain von Neumann subalgebras of $B(H)$. Our primary results give criteria by which it may be determined if two representations give rise to equal or conjugate endomorphisms.
\end{abstract}

\section{INTRODUCTION}

The connection between endomorphisms of factors and families of isometric operators has been explored most notably by Arveson [1, among others [2, 3, 6, 7]. In 6, Laca determines that given a normal $*$-endomorphism $\alpha$ of $B(H)$ there exists an $n \in \mathbb{N} \cup\{\infty\}$ and $*$-representation $\pi: \mathcal{E}_{n} \rightarrow B(H)$, where $\mathcal{E}_{n}$ denotes the Toeplitz algebra for $n$ orthogonal isometries $v_{1}, \ldots, v_{n}$, such that

$$
\alpha(T)=\sum_{i=1}^{n} \pi\left(v_{i}\right) T \pi\left(v_{i}\right)^{*}
$$

for each $T \in B(H)$. The $n$ value is unique but the representation $\pi$ may differ by automorphisms of $\mathcal{E}_{n}$ which arise specifically from unitary transformations of the Hilbert space $\ell^{2}\left(\left\{v_{1}, \ldots, v_{n}\right\}\right) \subseteq \mathcal{E}_{n}$ [6, Proposition 2.2].

In [2] and [3] Brenken extends the connection by determining that, for a von Neumann algebra which can be decomposed into a direct sum of Type I factors, a certain class of $*$-endomorphisms correspond to representations of certain $C^{*}$ algebras associated with (possibly infinite) matrices which arise as the adjacency matrices for directed graphs. The *-endomorphisms studied by Brenken are, however, required to either be unital [2] or satisfy a number of restrictive conditions [3, pp 25].

Our results extend the work of Brenken by eliminating the conditions imposed on the $*$-endomorphisms. The fundamental difference in our approach is that we will investigate representations of the Toeplitz algebra of a $C^{*}$-correspondence, whereas Brenken concerned himself with the so-called relative Cuntz-Pimnser algebras. We recover Brenken's results as a special case when the endomorphisms are assumed unital. Our primary result in this line is Theorem 4.1 .

2010 Mathematics Subject Classification. 46L05, 46L08, 46L55.

Key words and phrases. Toeplitz Algebras, Endomorphisms, $C^{*}$-correspondences, Graphs. 
Our results also continue the spirit of Laca's investigations which allow for equivalencies between $*$-endomorphisms to be encoded as transformations of an underlying linear object: a Hilbert space in the case of Laca and a $C^{*}$-correspondence in the present work. Our primary results along these lines are Theorems 3.2 and 3.4

\section{PRELIMINARIES}

First we will establish our terminology and notation.

Definition 1.1. A graph is a tuple $E=\left(E^{0}, E^{1}, r, s\right)$ consisting of a vertex set $E^{0}$, an edge set $E^{1}$, and range and source maps $r, s: E^{1} \rightarrow E^{0}$.

We will only consider graphs where $E^{0}$ and $E^{1}$ are at most countable.

Definition 1.2. Let $A$ be a $C^{*}$-algebra. A set $X$ is a $C^{*}$-correspondence over $A$ provided that it is a right Hilbert $A$-module and there is a $*$-homomorphism $\phi$ : $A \rightarrow L(X)$, where $L(X)$ denotes the space of adjointable $A$-module homomorphisms from $X$ to itself.

Given a $C^{*}$-correspondence $X$ over $A$, we will denote the $A$-valued inner product of $x, y \in X$ by " $\langle x, y\rangle_{A}$ " (perhaps omitting the $A$ ) and the right action of $a \in A$ on $x \in X$ will be written as " $x \cdot a$ ". The map $\phi: A \rightarrow L(X)$ may sometimes be written as $\phi_{X}$ for clarity.

Our primary objects of study will be certain $C^{*}$-correspondences which arise from graphs. The following constructions are due originally to Fowler and Raeburn [5. Example 1.2], although we will adopt the modern convention for the roles of $r$ and $s$.

Definition 1.3. Given a graph $E$, the graph correspondence $X(E)$ is the set of all functions $x: E^{1} \rightarrow \mathbb{C}$ for which $\hat{x}(v):=\sum_{e \in s^{-1}(v)}|x(e)|^{2}$ extends to a function $\hat{x} \in C_{0}\left(E^{0}\right)$. We give $X(E)$ the structure of a $C^{*}$-correspondence over $C_{0}\left(E^{0}\right)$ as follows:

$$
\begin{aligned}
x \cdot a: e & \mapsto x(e) a(s(e)), \\
\phi(a) x: e & \mapsto a(r(e)) x(e), \\
\langle x, y\rangle: v & \mapsto \sum_{e \in s^{-1}(v)} \overline{x(e)} y(e) .
\end{aligned}
$$

which is to say that $a \in C_{0}\left(E^{0}\right)$ acts on the right of $X(E)$ as multiplication by $a \circ s$ and acts on the left as multiplication by $a \circ r$.

The sets $\left\{\delta_{e}: e \in E^{1}\right\}$ and $\left\{\delta_{v}: v \in E^{0}\right\}$ are dense in $X(E)$ and $C_{0}\left(E^{0}\right)$, respectively, in the appropriate senses. For $e \in E^{1}$ and $v \in E^{0}$ we have the following useful relations: $\left\langle\delta_{e}, \delta_{e}\right\rangle=\delta_{s(e)}, \delta_{e} \cdot \delta_{v}=\delta_{e}$ if $v=s(e)$ and is 0 otherwise, and $\phi\left(\delta_{v}\right) \delta_{e}=\delta_{e}$ if $v=r(e)$ and is 0 otherwise.

Definition 1.4. Given a $C^{*}$-correspondence $X$ over $A$ and given another $C^{*}$ algebra $B$, a Toeplitz representation of $X$ in $B$ is a pair $(\sigma, \pi)$ consisting of a linear map $\sigma: X \rightarrow B$ and a $*$-homomorphism $\pi: A \rightarrow B$ such that for all $x, y \in X$ and $a \in A$

(i) $\sigma(x \cdot a)=\sigma(x) \pi(a)$,

(ii) $\sigma(\phi(a) x)=\pi(a) \sigma(x)$, and

(iii) $\pi(\langle x, y\rangle)=\sigma(x)^{*} \sigma(y)$. 
For a graph correspondence $X(E)$, a Toeplitz representation $(\sigma, \pi)$ is determined entirely by the values $\left\{\sigma\left(\delta_{e}\right): e \in E^{1}\right\}$ and $\left\{\pi\left(\delta_{v}\right): v \in E^{0}\right\}$. Property (iii) of a Toeplitz representation guarantees that $\sigma\left(\delta_{e}\right)$ is a partial isometry with source projection $\pi\left(\delta_{s(e)}\right)$.

Definition 1.5. [5, Proposition 1.3] Given a $C^{*}$-correspondence $X$ over $A$, the Toeplitz algebra of $X$, denoted $\mathcal{T}_{X}$, is the $C^{*}$-algebra which is universal in the following sense: there exists a Toeplitz representation $\left(\sigma_{u}, \pi_{u}\right)$ of $X$ in $\mathcal{T}_{X}$ such that if $(\sigma, \pi)$ is another Toeplitz representation of $X$ in a $C^{*}$-algebra $B$ then there exists a unique $*$-homomorphism $\rho_{\sigma, \pi}: \mathcal{T}_{X} \rightarrow B$ such that $\sigma=\rho_{\sigma, \pi} \circ \sigma_{u}$ and $\pi=\rho_{\sigma, \pi} \circ \pi_{u}$.

That $\mathcal{T}_{X}$ exists was proven by Pimnser in 9 .

Given a graph $E$ we may consider the Toeplitz algebra of its graph correspondence, cumbersomely denoted $\mathcal{T}_{X(E)}$. Unless there is danger of confusion, we will abuse notation and make no distinction between elements of $X(E)$ and $C_{0}\left(E^{0}\right)$ and their images in $\mathcal{T}_{X(E)}$ under the universal maps $\sigma_{u}$ and $\pi_{u}$.

If $\tau: \mathcal{T}_{X(E)} \rightarrow B(H)$ is a $*$-representation then, for each $e \in E^{1}, \tau\left(\delta_{e}\right)$ is a partial isometry with source projection $\tau\left(\delta_{s(e)}\right)$ and range projection contained in $\tau\left(\delta_{r(e)}\right)$.

If $E$ is the graph with but a single vertex and $n$ edges then $X(E)$ is a Hilbert space of dimension $n$ and $\mathcal{T}_{X(E)}$ is isomorphic to the classical Toeplitz algebra $\mathcal{E}_{n}$. In this case the elements $\left\{\delta_{e}: e \in E^{1}\right\}$ are precisely the generating isometries of $\mathcal{E}_{n}$. The space $X(E)$ plays a significant role in the analysis of endomorphisms of $B(H)$ in [6], and it is for this reason that we are considering the generalized Toeplitz algebras $\mathcal{T}_{X(E)}$ in our investigations.

\section{COHERENT UNITARY EQUIVALENCE}

Two graphs $E$ and $F$ are isomorphic if there are two bijections $\psi^{0}: E^{0} \rightarrow F^{0}$ and $\psi^{1}: E^{1} \rightarrow F^{1}$ for which $r_{F} \circ \psi^{1}=\psi^{0} \circ r_{E}$ and $s_{F} \circ \psi^{1}=\psi^{0} \circ s_{E}$. In order to encode such an isomorphism at the level of the graph correspondences $X(E)$ and $X(F)$, we offer the following novel definition.

Definition 2.1. Let $X$ and $Y$ be $C^{*}$-correspondences over $A$ and $B$, respectively. A coherent unitary equivalence between $X$ and $Y$ is a pair $(U, \alpha)$ consisting of a bijective linear map $U: X \rightarrow Y$ and a $*$-isomorphism $\alpha: A \rightarrow B$ for which

(i) $U(x \cdot a)=(U x) \cdot \alpha(a)$ for all $x \in X$ and $a \in A$,

(ii) $U\left(\phi_{X}(a) x\right)=\phi_{Y}(\alpha(a)) U x$ for all $x \in X$ and $a \in A$, and

(iii) $\langle U x, y\rangle_{Y}=\alpha\left(\left\langle x, U^{-1} y\right\rangle_{X}\right)$ for all $x \in X$ and $y \in Y$.

Routine calculations will verify that coherent unitary equivalence is an equivalence relation.

Proposition 2.2. If $E$ and $F$ are isomorphic graphs then $X(E)$ and $X(F)$ are coherently unitarily equivalent.

Proof. We'll assume $\left(\psi^{0}, \psi^{1}\right)$ to be an isomorphism from $F$ to $E$.

For $a \in C_{0}\left(E^{0}\right), \alpha(a):=a \circ \psi^{0}$ clearly defines a $*$-isomorphism $\alpha: C_{0}\left(E^{0}\right) \rightarrow$ $C_{0}\left(F^{0}\right)$. For $x \in X(E)$ define $U x:=x \circ \psi^{1}$. For $v \in F^{1}$ we have

$$
\sum_{e \in s_{F}^{-1}(v)}|U x(e)|^{2}=\sum_{e \in s_{F}^{-1}(v)}\left|x\left(\psi^{1}(e)\right)\right|^{2}=\sum_{f \in s_{E}^{-1}\left(\psi^{0}(v)\right)}|x(f)|^{2}
$$


(using the fact that if $s_{E}(e)=v$ then $\left.s_{F}\left(\psi^{1}(e)\right)=\psi^{0}(v)\right)$ and so $\widehat{U x}(v)=\hat{x}\left(\psi^{1}(v)\right)$. As $\hat{x} \in C_{0}\left(E^{0}\right)$ it follows immediately that $\widehat{U x} \in C_{0}\left(F^{0}\right)$, i.e. $U x \in X(F)$. Identical arguments show that $U^{-1} y:=y \circ\left(\psi^{1}\right)^{-1}$ is a map from $X(F)$ to $X(E)$ which is a two-sided inverse for $U$. Hence $U: X(E) \rightarrow X(F)$ is a bijection which is naturally linear.

Given $x \in X(E), a \in C_{0}\left(E^{0}\right)$, and $e \in E^{1}$ we have

$$
\begin{aligned}
U(x \cdot a) & =\left(x\left(a \circ s_{E}\right)\right) \circ \psi^{1}=\left(x \circ \psi^{1}\right)\left(a \circ s_{E} \circ \psi^{1}\right)=(U x)\left(a \circ \psi^{0} \circ s_{F}\right)=U x \cdot \alpha(a) \\
U\left(\phi_{E}(a) x\right) & =\left(\left(a \circ r_{E}\right) x\right) \circ \psi^{1}=\left(a \circ r_{E} \circ \psi^{1}\right)\left(x \circ \psi^{1}\right)=\left(a \circ \psi^{0} \circ r_{F}\right)(U x)=\phi_{F}(\alpha(a)) U x
\end{aligned}
$$

and, given $v \in F^{0}$,

$$
\begin{aligned}
\langle U x, y\rangle(v) & =\sum_{e \in s_{F}^{-1}(v)} \overline{U x(e)} y(e)=\sum_{e \in s_{F}^{-1}(v)} \overline{x\left(\psi^{1}(e)\right)} y(e)=\sum_{f \in s_{E}^{-1}\left(\psi^{0}(v)\right)} \overline{x(f)} y\left(\left(\psi^{1}\right)^{-1}(f)\right) \\
& =\sum_{f \in s_{E}^{-1}\left(\psi^{0}(v)\right)} \overline{x(f)} U^{-1} y(f)=\left\langle x, U^{-1} y\right\rangle\left(\psi^{0}(v)\right)=\alpha\left(\left\langle x, U^{-1} y\right\rangle\right)(v)
\end{aligned}
$$

(the first inner product is that of $X(F)$ and the later two are that of $X(E)$ ). Thus the pair of $U$ and $\alpha$ satisfies the definition of a coherent unitary equivalence.

Not every coherent unitary equivalence comes from a graph isomorphism in the sense of the preceding Proposition. As a simple example, consider the graph $E$ with but a single vertex and two edges. In this case $C_{0}\left(E^{0}\right)=\mathbb{C}$ and $X(E)=\mathbb{C}^{2}$. Hence any unitary $U \in M_{2}(\mathbb{C})$ forms (with the identify on $C_{0}\left(E^{0}\right)$ ) a coherent unitary equivalence. However, the only such equivalences arising from graph isomorphisms would be those of the two permutation matrices.

Proposition 2.3. If there is a coherent unitary equivalence between $X$ and $Y$ then $\mathcal{T}_{X}$ and $\mathcal{T}_{Y}$ are $*$-isomorphic.

Proof. Let $A$ and $B$ be the coefficient $C^{*}$-algebras for $X$ and $Y$, respectively. Suppose that $(U, \alpha)$ is a coherent unitary equivalence between $X$ and $Y$ and let $(\sigma, \pi)$ be a Toeplitz representation of $Y$. For $x \in X$ and $a \in A$

$$
\begin{aligned}
\sigma(U(x \cdot a)) & =\sigma(U x \alpha(a))=\sigma(U x) \pi(\alpha(a)) \\
\sigma\left(U\left(\phi_{X}(a) x\right)\right) & =\sigma(\alpha(a) U x)=\pi(\alpha(a)) \sigma(U x)
\end{aligned}
$$

and for $x_{1}, x_{2} \in X$

$$
\pi \circ \alpha\left(\left\langle x_{1}, x_{2}\right\rangle_{A}\right)=\pi\left(\left\langle U x_{1}, U x_{2}\right\rangle_{B}\right)=\sigma\left(U x_{1}\right)^{*} \sigma\left(U x_{2}\right) .
$$

Hence $(\sigma \circ U, \pi \circ \alpha)$ is a Toeplitz representation of $X$.

In particular, $\left(\sigma_{Y} \circ U, \pi_{B} \circ \alpha\right)$ is a Toeplitz representation of $X$ where $\left(\sigma_{Y}, \pi_{B}\right)$ is the universal Toeplitz representation of $Y$ in $\mathcal{T}_{Y}$. By the universal property of $\mathcal{T}_{X}$, there is a $*$-homomorphism $\theta: \mathcal{T}_{X} \rightarrow \mathcal{T}_{Y}$ such that $\theta \circ \sigma_{X}=\sigma_{Y} \circ U$ and $\theta \circ \pi_{A}=\pi_{B} \circ \alpha$, where $\left(\sigma_{X}, \pi_{A}\right)$ is the universal representation of $X$ in $\mathcal{T}_{X}$.

Similarly $\left(\sigma_{X} \circ U^{-1}, \pi_{A} \circ \alpha^{-1}\right)$ is a Toeplitz representation of $Y$ and induces a *-homomorphism $\theta^{\prime}: \mathcal{T}_{Y} \rightarrow \mathcal{T}_{X}$ for which $\theta^{\prime} \circ \sigma_{Y}=\sigma_{X} \circ U^{-1}$ and $\theta^{\prime} \circ \pi_{B}=\pi_{A} \circ \alpha^{-1}$. Thus

$$
\sigma_{Y}=\sigma_{Y} \circ U \circ U^{-1}=\theta \circ \sigma_{X} \circ U^{-1}=\theta \circ \theta^{\prime} \circ \sigma_{Y}
$$

and similarly $\pi_{B}=\theta \circ \theta^{\prime} \circ \pi_{B}$. Since the identity $i d$ on $\mathcal{T}_{Y}$ also has the property that $\pi_{B}=i d \circ \pi_{B}$ and $\sigma_{Y}=i d \circ \sigma_{Y}$, it follows by the universal property of $\mathcal{T}_{Y}$ that 
$\theta \circ \theta^{\prime}=i d$. Identical reasoning verifies that $\theta^{\prime} \circ \theta$ is the identity on $\mathcal{T}_{X}$. Thus $\theta$ is our desired $*$-isomorphism.

Going forward we will be exclusively interested in Toeplitz algebras associated to graph correspondences, and so offer the following corollary.

Corollary 2.4. Let $E$ and $F$ be graphs. If $(U, \alpha)$ is a coherent unitary equivalence between $X(E)$ and $X(F)$ then there is a $*$-isomorphism $\Gamma_{U, \alpha}: \mathcal{T}_{X(E)} \rightarrow \mathcal{T}_{X(F)}$ for which $\Gamma_{U, \alpha}\left(\delta_{e}\right)=U \delta_{e}$ and $\Gamma_{U, \alpha}\left(\delta_{v}\right)=\alpha\left(\delta_{v}\right)$ for all $e \in E^{1}$ and $v \in E^{0}$.

This is immediately seen from the proof of the previous Proposition if we recall that we identify $X(E)$ and $X(F)$ with their images in $\mathcal{T}_{X(E)}$ and $\mathcal{T}_{X(F)}$, respectively, under the appropriate universal maps. This also implies that if $E$ and $F$ are isomorphic graphs then $\mathcal{T}_{X(E)}$ and $\mathcal{T}_{X(F)}$ are $*$-isomorphic, which is unsurprising.

\section{ENDOMORPHISMS FROM GRAPHS}

Throughout this section we will let $E=\left(E^{0}, E^{1}, r, s\right)$ be a given graph whose vertex and edge sets are at most countable. All $*$-representations will be assumed non-degenerate.

Proposition 3.1. Given a $*$-representation $\tau: \mathcal{T}_{X(E)} \rightarrow B(H)$, the assignments

$$
A d_{\tau}(w):=\sum_{e \in E^{1}} \tau\left(\delta_{e}\right) w \tau\left(\delta_{e}\right)^{*}
$$

(the sum is taken as a SOT limit) define a *-endomorphism $A d_{\tau}$ of the von Neumann algebra $W=\left\{\tau\left(\delta_{v}\right): v \in E^{0}\right\}^{\prime}$ (this notation will denote the relative commutant in $B(H))$.

Proof. First, notice that for $e \in E^{1}$ and $w \in W$ the term $\tau\left(\delta_{e}\right) w \tau\left(\delta_{e}\right)^{*}$ has its support projection contained in $\tau\left(\delta_{e}^{*} \delta_{e}\right)$. Since the partial isometries $\tau\left(\delta_{e}\right)$ have mutually orthogonal ranges, it follows that for every $h \in H, \tau\left(\delta_{e}\right) w \tau\left(\delta_{e}\right)^{*} h$ is nonzero for at most one $e \in E^{1}$. Thus the sum converges in the SOT.

Certainly $A d_{\tau}$ is linear and has $A d_{\tau}\left(w^{*}\right)=A d_{\tau}(w)^{*}$ for each $w \in W$. Given $w_{1}, w_{2} \in W$ we find that

$$
\begin{aligned}
A d_{\tau}\left(w_{1}\right) A d_{\tau}\left(w_{2}\right) & =\left(\sum_{e \in E^{1}} \tau\left(\delta_{e}\right) w_{1} \tau\left(\delta_{e}\right)^{*}\right)\left(\sum_{f \in E^{1}} \tau\left(\delta_{f}\right) w_{2} \tau\left(\delta_{f}\right)^{*}\right) \\
& =\sum_{e, f \in E^{1}} \tau\left(\delta_{e}\right) w_{1} \tau\left(\delta_{e}\right)^{*} \tau\left(\delta_{f}\right) w_{2} \tau\left(\delta_{f}\right)^{*} \\
& =\sum_{e \in E^{1}} \tau\left(\delta_{e}\right) w_{1} \tau\left(\delta_{s(e)}\right) w_{2} \tau\left(\delta_{e}\right)^{*} \\
& =\sum_{e \in E^{1}} \tau\left(\delta_{e}\right) \tau\left(\delta_{s(e)}\right) w_{1} w_{2} \tau\left(\delta_{e}\right)^{*} \\
& =\sum_{e \in E^{1}} \tau\left(\delta_{e}\right) w_{1} w_{2} \tau\left(\delta_{e}\right)^{*} \\
& =A d_{\tau}\left(w_{1} w_{2}\right)
\end{aligned}
$$

and so $A d_{\tau}$ is multiplicative. Note that any potential issues with SOT-convergence of the product are circumvented by $E^{1}$ being at most countable. All that remains is to verify that $A d_{\tau}(w) \in W$ for each $w \in W$. To that end we first note that 
$\delta_{e}^{*} \delta_{v}=\delta_{e}^{*}$ if $v=r(e)$ and is zero otherwise. By taking adjoints, $\delta_{v} \delta_{e}=\delta_{e}$ if $v=r(e)$ and is zero otherwise. Thus, given $w \in W$ and $v \in E^{0}$ we find

$$
A d_{\tau}(w) \tau\left(\delta_{v}\right)=\sum_{e \in r^{-1}(v)} \tau\left(\delta_{e}\right) w \tau\left(\delta_{e}\right)^{*}=\tau\left(\delta_{v}\right) A d_{\tau}(w)
$$

and so $A d_{\tau}(w)$ commutes with each $\tau\left(\delta_{v}\right)$.

We note that the von Neumann algebra $W=\left\{\tau\left(\delta_{v}\right): v \in E^{0}\right\}$, because the $\tau\left(\delta_{v}\right)$ are a family of mutually orthogonal projections, is precisely equal to $\bigoplus_{v \in E^{0}} \tau\left(\delta_{v}\right) B(H) \tau\left(\delta_{v}\right)$ which is a sum of Type I factors.

The following is a construction which we believe to be folklore, but our use of it is motivated by observations made by Muhly and Solel [8]. Given a $*$-representation $\tau: \mathcal{T}_{X(E)} \rightarrow B(H)$ let $W=\left\{\tau\left(\delta_{v}\right): v \in E^{0}\right\}^{\prime}$. The space

$$
\mathcal{I}_{\tau}:=\left\{T \in B(H): A d_{\tau}(w) T=T w, w \in W\right\}
$$

is a $C^{*}$-correspondence over $W^{\prime}$. The left and right actions of $W^{\prime}$ are simply multiplication within $B(H)$ and the $W^{\prime}$-valued inner product is defined by $\langle T, S\rangle_{W^{\prime}}:=$ $T^{*} S$.

Because our endomorphism is of the form $A d_{\tau}$, we can say more: for $w \in W$ and $e \in E^{1}$

$A d_{\tau}(w) \tau\left(\delta_{e}\right)=\sum_{f \in E^{1}} \tau\left(\delta_{f}\right) w \tau\left(\delta_{f}\right)^{*} \tau\left(\delta_{e}\right)=\tau\left(\delta_{e}\right) w \tau\left(\delta_{s(e)}\right)=\tau\left(\delta_{e}\right) \tau\left(\delta_{s(e)}\right) w=\tau\left(\delta_{e}\right) w$

and so $\tau\left(\delta_{e}\right) \in \mathcal{I}_{\tau}$ for each $e \in E^{1}$. As $\tau\left(\delta_{v}\right) \in W^{\prime}$ for each $v \in E^{0}$ we finally have $\tau(X(E)) \subseteq \mathcal{I}_{\tau}$.

Theorem 3.2. Suppose that $E$ and $F$ are graphs and $\tau_{1}: \mathcal{T}_{X(E)} \rightarrow B(H)$ and $\tau_{2}: \mathcal{T}_{X(F)} \rightarrow B(H)$ are two faithful $*$-representations. If $A d_{\tau_{1}}=A d_{\tau_{2}}$ on $W=$ $\left\{\tau_{1}\left(\delta_{v}\right): v \in E^{0}\right\}^{\prime}=\left\{\tau_{2}\left(\delta_{v}\right): v \in F^{0}\right\}^{\prime}$ then there is a coherent unitary equivalence $(U, \alpha)$ between $X(E)$ and $X(F)$ such that $\tau_{2}=\tau_{1} \circ \Gamma_{U, \alpha}$.

Here $\Gamma_{U, \alpha}$ is the $*$-isomorphism from $\mathcal{T}_{X(E)}$ to $\mathcal{T}_{X(F)}$ arising from $(U, \alpha)$ as given in Corollary 2.4

Proof. Since $\left\{\tau_{1}\left(\delta_{v}\right): v \in E^{0}\right\}$ and $\left\{\tau_{2}\left(\delta_{v}\right): v \in F^{0}\right\}$ are sets of orthogonal projections with the same commutant they are in fact equal, and in particular $E^{0}$ and $F^{0}$ are of the same cardinality. To ease notation we'll denote these projections by $P_{v}, v \in E^{0}$, (with no assumption that $P_{v}=\tau_{1}\left(\delta_{v}\right)$ or similar) hence

$$
\left\{P_{v}: v \in E^{0}\right\}=\left\{\tau_{1}\left(\delta_{v}\right): v \in E^{0}\right\}=\left\{\tau_{2}\left(\delta_{v}\right): v \in F^{0}\right\} .
$$

As $A d_{\tau_{1}}=A d_{\tau_{2}}$ we have that $\mathcal{I}_{\tau_{1}}=\mathcal{I}_{\tau_{2}}$ and we'll call this module simply $\mathcal{I}$.

As $\tau_{1}\left(\delta_{e}\right) \in \mathcal{I}$ for each $e \in E^{1}$ we have

$$
\tau_{1}\left(\delta_{e}\right)=\tau_{1}\left(\delta_{e}\right) I=A d_{\tau_{2}}(I) \tau_{1}\left(\delta_{e}\right)=\sum_{f \in F^{1}} \tau_{2}\left(\delta_{f}\right) \tau_{2}\left(\delta_{f}\right)^{*} \tau_{1}\left(\delta_{e}\right)
$$

hence $\tau_{1}\left(\delta_{e}\right)$ is in the $W^{\prime}$-submodule of $\mathcal{I}$ generated by $\tau_{2}(X(E))$. Similarly, for each $f \in F^{1}, \tau_{2}\left(\delta_{f}\right)$ is in the $W^{\prime}$-submodule generated by $\tau_{1}(X(E))$. Thus $\tau_{1}(X(E))$ and $\tau_{2}(X(E))$ generate the same $W^{\prime}$-submodule of $\mathcal{I}$.

Given $e \in E^{1}$ and $f \in F^{1}$ we have seen that

$$
\tau_{2}\left(\delta_{f}\right)^{*} \tau_{1}\left(\delta_{e}\right) \in W^{\prime}=\left\{P_{v}: v \in E^{0}\right\}^{\prime \prime}=\ell^{\infty}\left(\left\{P_{v}: v \in E^{0}\right\}\right) .
$$


Notice however that $\tau_{2}\left(\delta_{f}\right)^{*} \tau_{1}\left(\delta_{e}\right) \tau_{1}\left(\delta_{v}\right)=0$ unless $v=s(e)$ and hence $\tau_{2}\left(\delta_{f}\right)^{*} \tau_{1}\left(\delta_{e}\right)$ is a multiple of $\tau_{1}\left(\delta_{s(e)}\right)$ only, i.e. is an element of $C_{0}\left(\left\{P_{v}: v \in E^{0}\right\}\right)$. Since before we obtained $\tau_{1}\left(\delta_{e}\right)=\sum_{f \in F^{1}} \tau_{2}\left(\delta_{f}\right) \tau_{2}\left(\delta_{f}\right)^{*} \tau_{1}\left(\delta_{e}\right)$ for all $e \in E^{1}$, it now follows that $\tau_{1}(X(E))$ and $\tau_{2}(X(E))$ generate the same correspondence over $C_{0}\left(\left\{P_{v}: v \in\right.\right.$ $\left.\left.E^{0}\right\}\right)$. It is important to note that this correspondence has three different actions of $C_{0}\left(\left\{P_{v}: v \in E^{0}\right\}\right)$ : the ones inherited through $\tau_{1}$ and $\tau_{2}$ and simple operator multiplication in $B(H)$.

Finally we have that $\tau_{1}\left(C_{0}\left(E^{0}\right)\right)=\tau_{2}\left(C_{0}\left(F^{0}\right)\right)$ and $\tau_{1}(X(E))=\tau_{2}(X(F))$ as sets and so, because both representations are faithful by hypothesis, $\tau_{2}^{-1} \circ \tau_{1}$ is a well-defined bijection between $X(E)$ and $X(F)$ and between $C_{0}\left(E^{0}\right)$ and $C_{0}\left(F^{0}\right)$. Denote by $U$ and $\alpha$ the restrictions of $\tau_{2}^{-1} \circ \tau_{1}$ to $X(E)$ and to $C_{0}\left(E^{0}\right)$, respectively.

Given $x \in X(E), y \in X(F)$, and $a \in C_{0}\left(E^{0}\right)$ we have

$$
\begin{aligned}
U(x a) & =\tau_{2}^{-1} \circ \tau_{1}(x a)=\tau_{2}^{-1} \circ \tau_{1}(x) \tau_{2}^{-1} \circ \tau_{1}(a)=(U x) \alpha(a), \\
U(\phi(a) x) & =\tau_{2}^{-1} \circ \tau_{1}(\phi(a) x)=\tau_{2}^{-1} \circ \tau_{1}(a) \tau_{2}^{-1} \circ \tau_{1}(x)=\alpha(a) U x, \\
\langle U x, y\rangle & =\left[\tau_{2}^{-1} \circ \tau_{1}(x)\right]^{*} y=\tau_{2}^{-1} \circ \tau_{1}\left(x^{*} \tau_{1}^{-1} \circ \tau_{2}(y)\right)=\alpha\left(\left\langle x, \tau_{1}^{-1} \circ \tau_{2}(y)\right\rangle\right)=\alpha\left(\left\langle x, U^{-1} y\right\rangle\right) .
\end{aligned}
$$

and so $(U, \alpha)$ is a coherent unitary equivalence between $X(E)$ and itself.

It follows from Corollary 2.4 that $(U, \alpha)$ induces a $*$-isomorphism $\Gamma_{U, \alpha}: \mathcal{T}_{X(E)} \rightarrow$ $\mathcal{T}_{X(F)}$ and, by construction, $\tau_{2} \circ \Gamma_{U, \alpha}=\tau_{1}$.

Notice we have shown that if $\tau_{1}: \mathcal{T}_{X(E)} \rightarrow B(H)$ and $\tau_{2}: \mathcal{T}_{X(F)} \rightarrow B(H)$ generate identical *-endomorphims $A d_{\tau_{1}}$ and $A d_{\tau_{2}}$ then $\mathcal{T}_{X(E)}$ and $\mathcal{T}_{X(F)}$ are *isomorphic as $C^{*}$-algebras. Hence in subsequent results we will concern ourselves only with two representations $\tau_{1}, \tau_{2}$ of the same Toeplitz algebra $\mathcal{T}_{X(E)}$.

Our result is a generalization of Laca's [6, Proposition 2.2]. When $E$ is the graph with a single vertex and $n \in \mathbb{N} \cup\{\infty\}$ edges we have already seen that $\mathcal{T}_{X(E)}=\mathcal{E}_{n}$. If $\tau_{1}$ and $\tau_{2}$ are faithful and nondegenerate then $W=B(H)$. The map $\alpha$ is the identity on $C_{0}\left(E^{0}\right)=\mathbb{C}$ and $U$ is a unitary operator on the Hilbert space $X(E)=$ $\ell^{2}\left(\left\{v_{1}, \ldots, v_{n}\right\}\right)$. Hence $\Gamma_{U, \alpha}$ is a $*$-automorphism of $\mathcal{E}_{n}$ which fixes the Hilbert space $X(E)$ and implements the equivalence between the two representations.

We will conclude this section with a discussion of conjugacy conditions for endomorphisms of the type we've been examining. Recall that two endomorphisms $\alpha$ and $\beta$ are said to be conjugate if there is an automorphism $\gamma$ such that $\alpha \circ \gamma=\gamma \circ \beta$.

Lemma 3.3. If $P_{1}, P_{2}, \ldots \in B(H)$ is an at most countable family of orthogonal projections and $\gamma$ is a *-automorphism of $W=\left\{P_{1}, P_{2}, \ldots\right\}^{\prime}$ then there exists a unitary $U \in B(H)$ such that $\gamma(w)=U w U^{*}$ for all $w \in W$.

Proof. Note that for each $n, \gamma$ restricts to a $*$-isomorphism $\gamma_{n}$ between $P_{n} B(H) P_{n}=$ $B\left(P_{n} H\right)$ and $\gamma\left(P_{n}\right) B(H) \gamma\left(P_{n}\right)=B\left(\gamma\left(P_{n}\right) H\right)$. Such isomorphisms are always spatial and so there are unitaries $U_{n}: B\left(P_{n} H\right) \rightarrow B\left(\gamma\left(P_{n}\right) H\right)$ such that $\gamma_{n}(w)=$ $U_{n} w U_{n}^{*}$. It is then immediate that $U:=U_{1} \oplus U_{2} \oplus \ldots$ is a unitary in $B(H)$ and $U w U^{*}=\gamma(w)$ for each $w \in W$.

Theorem 3.4. Suppose that $\tau_{1}, \tau_{2}: \mathcal{T}_{X(E)} \rightarrow B(H)$ are two faithful $*$-representations such that $A d_{\tau_{1}}$ and $A d_{\tau_{2}}$ are conjugate $*$-endomorphisms of $W=\left\{\tau_{1}\left(\delta_{v}\right): v \in\right.$ $\left.E^{0}\right\}^{\prime}=\left\{\tau_{2}\left(\delta_{v}\right): v \in E^{0}\right\}^{\prime}$. Then there is a coherent unitary equivalence $(U, \alpha)$ between $X(E)$ and itself such that $\tau_{2}$ and $\tau_{1} \circ \Gamma_{U, \alpha}$ are unitarily equivalent $*$ representations. 
Proof. Let $\gamma$ be an $*$-automorphism of $W$ such that $A d_{\tau_{1}} \circ \gamma=\gamma \circ A d_{\tau_{2}}$ and let $V \in B(H)$ be the unitary for which $\gamma(w)=V w V^{*}$ according the Lemma 3.3. Then $A d_{\tau_{2}}(w)=V^{*} A d_{\tau_{1}}\left(V w V^{*}\right) V$ for all $w \in W$. Define $\kappa: \mathcal{T}_{X(E)} \rightarrow B(H)$ by $\kappa(t):=V \tau_{1}(t) V^{*}$ and note that $\kappa$ is a $*$-representation of $\mathcal{T}_{X(E)}$ such that

$$
A d_{\kappa}(w)=\sum_{e \in E^{1}} \kappa\left(\delta_{e}\right) w \kappa\left(\delta_{e}\right)^{*}=\sum_{e \in E^{1}} V \tau_{1}\left(\delta_{e}\right) V^{*} w V \tau_{1}\left(\delta_{e}\right)^{*} V^{*}=V A d_{\tau_{1}}\left(V^{*} w V\right) V^{*}
$$

and so $A d_{\kappa}=A d_{\tau_{2}}$ on $W$. Applying Theorem 3.2 we obtain a coherent unitary equivalence $(U, \alpha)$ inducing the $*$-automorphism $\Gamma_{U, \alpha}$ of $\mathcal{T}_{X(E)}$ such that $\tau_{2}=$ $\kappa \circ \Gamma_{U, \alpha}$. As now $\tau_{2}(t)=V\left[\tau_{1} \circ \Gamma_{U, \alpha}(t)\right] V^{*}$ for each $t \in \mathcal{T}_{X(E)}$, we have that $\tau_{2}$ and $\tau_{1} \circ \Gamma_{U, \alpha}$ are unitarily equivalent, as desired.

\section{GRAPHS FROM ENDOMORPHISMS}

In this final section we will demonstrate that all $*$-endomorphisms of von Neumann algebras which are sums of Type I factors are obtained in the natural way from representations of Toeplitz algebras for graph correspondences. Our result is a significant generalization of [3, Theorem 3.9] which places technical restrictions on the endomorphisms. In the case of unital endomorphisms our results are comparable.

Theorem 4.1. Let $W=\bigoplus W_{i} \subseteq B(H)$ be a countable sum of Type I factors. Let $P_{1}, P_{2}, \ldots \in B(H)$ be projections such that $W_{i}=P_{i} B(H) P_{i}$. If $\alpha$ is a normal $*$ endomorphism of $W$ then there exists a graph $E$ and $*$-representation $\tau: \mathcal{T}_{X(E)} \rightarrow$ $B(H)$ such that $\alpha=A d_{\tau}$.

Proof. Without loss of generality we may assume that $\sum P_{i}=I$. If this were not the case we may define $P_{0}=\left(\sum P_{i}\right)^{\perp}$ and $W_{0}=P_{0} B(H) P_{0}$ and extend $\alpha$ to a normal $*$-endomorphism of $W \oplus W_{0}$ with $\left.\alpha\right|_{W_{0}}=i d$.

For $i>0$ define $H_{i}=P_{i} H$. For $i, j>0$ and $x \in W$ define $\alpha_{i j}(x)=P_{j} \alpha\left(P_{i} x\right)$. Then $\alpha_{i j}$ restricts to a $*$-homomorphism between $B\left(H_{i}\right)=P_{i} B(H) P_{i}=W_{i}$ and $B\left(H_{j}\right)=P_{j} B(H) P_{j}=W_{j}$ as seen by

$$
P_{j} \alpha\left(P_{i} x\right) P_{j} \alpha\left(P_{i} y\right)=P_{j}\left(P_{j} \alpha\left(P_{i} x\right)\right) \alpha\left(P_{i} y\right)=P_{j} \alpha\left(P_{i} x P_{i} y\right)=P_{j} \alpha\left(P_{i} x y\right) .
$$

So $\alpha_{i j}$ is a $*$-homomorphism between two Type I factors, and thus by [1, Proposition 2.1] if $\alpha_{i j}$ is nonzero there exists $n_{i j} \in \mathbb{N} \cup\{\infty\}$ and isometries $V_{k}^{(i j)} \in$ $B\left(H_{i}, H_{j}\right), k=1, \ldots, n_{i j}$ such that $\left.\alpha_{i j}\right|_{B\left(H_{i}\right)}(T)=\sum_{k=1}^{n_{i j}} V_{k}^{(i j)} T V_{k}^{(i j) *}$. We will identify the $V_{k}^{(i j)}$ with their associated partial isometries in $B(H)$, so that $V_{k}^{(i j) *} V_{k}^{(i j)}=$ $P_{i}$ and $V_{k}^{(i j)} V_{k}^{(i j) *} \leq P_{j}$.

Set $E^{0}:=\left\{P_{1}, P_{2} \ldots,\right\}$ and $E^{1}:=\bigcup_{i, j}\left\{V_{k}^{(i j)}: k=1, \ldots, n_{i j}\right\}$. Define maps $r, s: E^{1} \rightarrow E^{0}$ by $r\left(V_{k}^{(i j)}\right)=P_{j}$ and $s\left(V_{k}^{(i j)}\right)=P_{i}$. Then $E=\left(E^{0}, E^{1}, r, s\right)$ is a graph. It is trivial to see that the identity maps on $E^{1}$ and $E^{0}$ extend to a Toeplitz representation of $X(E)$ which in turn induces a $*$-representation $\tau: \mathcal{T}_{X(E)} \rightarrow B(H)$.

Finally, we have that for each $x \in W$

$$
\alpha(x)=\sum_{i, j>0} P_{j} \alpha\left(P_{i} x\right)=\sum_{i, j>0} \alpha_{i j}(x)=\sum_{i, j>0} \sum_{k=1}^{n_{i j}} V_{k}^{(i j)} x V_{k}^{(i j) *}=\sum_{f \in E^{1}} \tau\left(\delta_{f}\right) x \tau\left(\delta_{f}\right)^{*}
$$

as desired. 
As a consequence, the possible $*$-endomorphisms of a given von Neumann algebra $W=\left\{P_{1}, P_{2}, \ldots\right\}^{\prime}$ coincide, up to conjugacy, with the coherent unitary equivalence classes of $C^{*}$-correspondences over $C_{0}\left(\left\{P_{1}, P_{2}, \ldots\right\}\right)$.

4.1. Cuntz-Pimsner Algebras. To discuss the special case of unital $*$-endomorphisms we will need to briefly sketch the defining features and universal properties of the so-called Cuntz-Pimsner algebras. The relationship of these Cuntz-Pimnser algebras to our Toeplitz algebras is analogous to the relationship between the classical Toeplitz algebras $\mathcal{E}_{n}$ and classical Cuntz algebras $\mathcal{O}_{n}$. The Cuntz-Pimsner algebras were originally defined in [9] though our treatment will take its cues from [4] and [5].

Recalling that $X(E)$ is a $C^{*}$-correspondence over $C_{0}\left(E^{0}\right)$, notice that $I=$ $C_{0}\left(\left\{v \in E^{0}: r^{-1}(v)\right.\right.$ is finite $\left.\}\right)$ is a closed, two-sided ideal in $C_{0}\left(E^{0}\right)$. As noted in [5. Proposition 4.4] (and recalling our modern reversal of $r$ and $s$ from the presentation in that work), elements of $I$ are precisely those elements of $C_{0}\left(E^{0}\right)$ whose left action on $X(E)$ is compact. Since our graphs have at most countable vertices and edges, $X(E)$ is countably generated as a $C^{*}$-correspondence over $C_{0}\left(E^{0}\right)$. It follows, [9, Remark 3.9 following Definition 3.8] that the Cuntz-Pimnser algebra of $X(E)$ is the $C^{*}$-algebra $\mathcal{O}_{X(E)}$ which is universal for Toeplitz representations $(\sigma, \pi)$ (as in Definition 1.4) which additionally satisfy:

(iv) $\pi(a)=\sum_{f \in E^{1}} \sigma\left(\phi(a) \delta_{f}\right) \sigma\left(\delta_{f}\right)^{*}$ for all $a \in I$.

or, equivalently (see [4, Example 1.5]),

(iv) $)^{\prime} \pi\left(\delta_{v}\right)=\sum_{f \in r^{-1}(v)} \sigma\left(\delta_{f}\right) \sigma\left(\delta_{f}\right)^{*}$ for all $v \in E^{0}$ with $\left|r^{-1}(v)\right|<\infty$.

Representations satisfying (i)-(iv)' are often termed "coisometric" Toeplitz representations. With this characterization of $\mathcal{O}_{X(E)}$ we may recognize that Theorem 4.1 has more to say when the endomorphism is unital.

Corollary 4.2. Let $W=\bigoplus W_{i} \subseteq B(H)$ be a countable sum of Type I factors. Let $P_{1}, P_{2}, \ldots \in B(H)$ be projections such that $W_{i}=P_{i} B(H) P_{i}$. If $\alpha$ is a normal, unital $*$-endomorphism of $W$ then there exists a graph $E$ and $*$-representation $\tau$ : $\mathcal{O}_{X(E)} \rightarrow B(H)$ such that $\alpha=A d_{\tau}$.

Proof. We define the partial isometries $V_{k}^{(i j)}$ and graph $E$ in the same manner as in the proof of Theorem 4.1. Since $\alpha$ is unital we have

$$
P_{n}=P_{n} \alpha(I)=\sum_{i, j>0} \sum_{k=1}^{n_{i j}} P_{n} V_{k}^{(i j)} V_{k}^{(i j) *}=\sum_{i>0} \sum_{k=1}^{n_{i n}} V_{k}^{(i n)} V_{k}^{(i n) *}
$$

for every $P_{n}$. Recalling that the representation of $(\sigma, \pi) E$ on $B(H)$ is the pair of identity maps, this becomes

$$
\pi\left(\delta_{v}\right)=\sum_{f \in E^{1}} \pi\left(\delta_{v}\right) \sigma\left(\delta_{f}\right) \sigma\left(\delta_{f}\right)^{*}=\sum_{f \in r^{-1}(v)} \sigma\left(\delta_{f}\right) \sigma\left(\delta_{f}\right)^{*}
$$

for all $v \in E^{0}$. In particular this holds for all edges $v$ with $r^{-1}(v)$ finite (i.e. all $P_{j}$ such that $\left\{V_{k}^{(i j)}: i>0, k=1, \ldots, n_{i j}\right\}$ is finite). Thus condition (iv) ${ }^{\prime}$ is satisfied and the identity maps on $E^{0}$ and $E^{1}$ induce a representation of $\mathcal{O}_{X(E)}$. The fact that $\alpha=A d_{\tau}$ follows as before. 


\section{REFERENCES}

[1] William Arveson. Continuous analogues of Fock space. Mem. Amer. Math. Soc., 80(409):iv+66, 1989.

[2] Berndt Brenken. Cuntz-Krieger algebras and endomorphisms of finite direct sums of type $\mathrm{I}_{\infty}$ factors. Trans. Amer. Math. Soc., 353(10):3835-3873, 2001.

[3] Berndt Brenken. Endomorphisms of type I von Neumann algebras with discrete center. $J$. Operator Theory, 51(1):19-34, 2004.

[4] Neal J. Fowler, Paul S. Muhly, and Iain Raeburn. Representations of Cuntz-Pimsner algebras. Indiana Univ. Math. J., 52(3):569-605, 2003.

[5] Neal J. Fowler and Iain Raeburn. The Toeplitz algebra of a Hilbert bimodule. Indiana Univ. Math. J., 48(1):155-181, 1999.

[6] M. Laca. Endomorphisms of $B(H)$ and Cuntz algebras. J. Operator Theory, 30(1):85-108, 1993.

[7] Roberto Longo. Simple injective subfactors. Adv. in Math., 63(2):152-171, 1987.

[8] Paul S. Muhly and Baruch Solel. Quantum Markov processes (correspondences and dilations). Internat. J. Math., 13(8):863-906, 2002.

[9] Michael V. Pimsner. A class of $C^{*}$-algebras generalizing both Cuntz-Krieger algebras and crossed products by Z. In Free probability theory (Waterloo, ON, 1995), volume 12 of Fields Inst. Commun., pages 189-212. Amer. Math. Soc., Providence, RI, 1997.

Department of Mathematics, State University of New York College at Cortland, Cortland, NY 13045-0900

E-mail address: philip.gipson@cortland.edu 